\title{
A technique to determine systematic shifts in microseismic databases
}

\author{
IG Morkel Australian Centre for Geomechanics and The University of Western Australia, Australia \\ J Wesseloo Australian Centre for Geomechanics and The University of Western Australia, Australia
}

\begin{abstract}
Due to the complex nature of the seismic response to mining, geotechnical engineers often require back analysis to provide a base line against which to interpret future behaviour. This practice assumes, and is reliant on, the database being consistent in space and time. Few tools are available for geotechnical engineers dedicated to the task of quantifying the consistency of the seismic database, and to aid in identifying systematic inconsistencies in their databases. A methodical approach is also required to warn geotechnical engineers of unexpected systematic shifts in their database as soon as they arise so that timeous and appropriate action can be taken. The industry collectively also requires a systematic approach to quantify the consistency of seismic databases.

A technique is proposed to adequately address these aims. The technique is fast and efficient and can be easily employed on any database. By continuously updating results, users would know within a few tens to hundreds of events when data shifts have occurred. This would allow for the effective management of these errors in the database.

Application of the method on some current industry databases showed that the shifts are sufficiently significant to render the use of some widely used analysis techniques unreliable. It is shown that shifts in the data have a significant influence on the interpretation of the source parameters.

Systematic errors are causing significant artefacts in seismic databases. Of the 20 databases investigated, $70 \%$ had one or more systematic shifts a year, and only one database showed no shifts at all. There is justified concern with respect to systematic inconsistencies in seismic databases in the industry. Such inconsistencies could lead to misinterpretation of seismic analysis results, which will have a carry-on effect on other parts of the operations.
\end{abstract}

Keywords: data quality, data consistency, seismic analysis, mine seismology

\section{Introduction}

It is well-known and generally accepted that data driven decisions are highly dependent on data quality and the slogan 'garbage in - garbage out' has become somewhat of a cliché. This is of course also for geotechnical data and seismic data in particular, which is the focus of this study.

The occurrence of seismicity in high stress hard rock mines poses a challenge to geotechnical engineers around the world who needs to base decisions on recorded seismic data. Potvin (2009) discusses the use of tactical and strategic approaches for the mitigation of seismic risk. These approaches include dynamic support design to prevent failure, mine layouts to minimise stress concentrations, and re-entry protocols to limit exposure during times of increased hazard. Using a combination of these approaches is necessary for the effective mitigation of seismic risk. Potvin (2009) describes the seismic risk as the combination of seismic hazard, exposure to the hazard, and the ability to effectively control the hazard. A reliable assessment of the seismic hazard in space and time is a crucial component of the assessment and management of seismic risk in mines. Currently, many analysis tools exist to evaluate the risk associated with seismicity. Many of these tools rely heavily on the use of seismic source parameters. 
A small sample of these studies includes:

- Alcott et al. (1998) discuss the use of seismic source parameters for assessing the hazard of rockbursts.

- Van Aswegen (2005) shows how changes in source parameters can be used for calculating routine short term seismic hazards.

- In a more recent study, Rebuli and Van Aswegen (2013) added to the Van Aswegen (2005) work additional parameters determined from mine production data.

- Scheepers et al. (2012) employed the use of seismic potency to develop a strategy with which to model the expected seismic response for future design.

- Wesseloo (2013) discusses the calculation of seismic hazard that relies on magnitude, which is determined from seismic moment, seismic energy or the combination of them.

- Wesseloo (2014) and Wesseloo et al. (2014) use the spatial properties of seismic source parameters and $b$-values to interpret the rock mass response.

- Disley (2014) describes the use of source parameters for re-entry protocol at the Kidd Creek Mine in Canada.

- Brown (2015) and Brown et al. (2015) show how apparent stress could be used to assess the seismic hazard in Canadian mines.

All these techniques rely on the consistency of the seismic source parameters for a system. It is important that the data used for any source parameter analyses are consistent.

Due to the complex nature of the seismic response to mining, geotechnical engineers often require back analysis to provide a base line against which to interpret future behaviour. This practice assumes, and is utterly reliant on, the database being consistent in space and time. Few tools are available for geotechnical engineers dedicated to the task of quantifying and investigating the consistency of their seismic database. A methodical approach is also required to warn geotechnical engineers of unexpected systematic shifts in their database as soon as it arises. This will ensure that timeous and appropriate action can be taken. The industry collectively also requires a systematic approach to quantify the consistency of seismic databases.

This paper provides a simple, effective and computationally efficient technique for identifying database inconsistencies and to quantify the extent of those inconsistencies. A detailed case study shows the application of the technique to a mining database and also the comparative results from several sites across the world.

\section{Complexity in the seismic system}

The recording of seismicity is a complex process. There are numerous known and unknown factors and assumptions that influence the final calculated source parameters. These include the system settings, human influence through processing decisions, algorithms and the natural variability in the rock mass, as well as the presence of mining voids.

Figure 1 illustrates the long chain of events involved when recording seismic events. The seismic waves propagate through the rock mass with many known and unknown defects and voids, all reflecting and refracting the waves resulting in a complex wave signal recorded at each of the sensors. Different sensors (accelerometers or geophones) with different frequency ranges record these complex waves and each of these sensors then impose its own bias onto the data (Hanks 1982). This sensor bias includes the sensor response function but also, for example, inaccuracies in the installation orientation or sensor-grout-rock bond. Some data loss may occur through triggering and association settings used on the system. Other errors may be introduced through inaccuracy or inconsistency in system configuration. Human influence also has an effect on the results of the system when manual processing is performed. Although some automatic location processing algorithms are available and show promise, manual processing is still common practice 
and, in some cases such as the processing of complex events, desirable. Different algorithms may result in different results both for location and the determination of source parameters. Systematic errors can propagate and accumulate through the system. Considering this complex chain of events, it is clear that ensuring the quality of the seismic database is not a trivial task and requires dedication from all stakeholders.

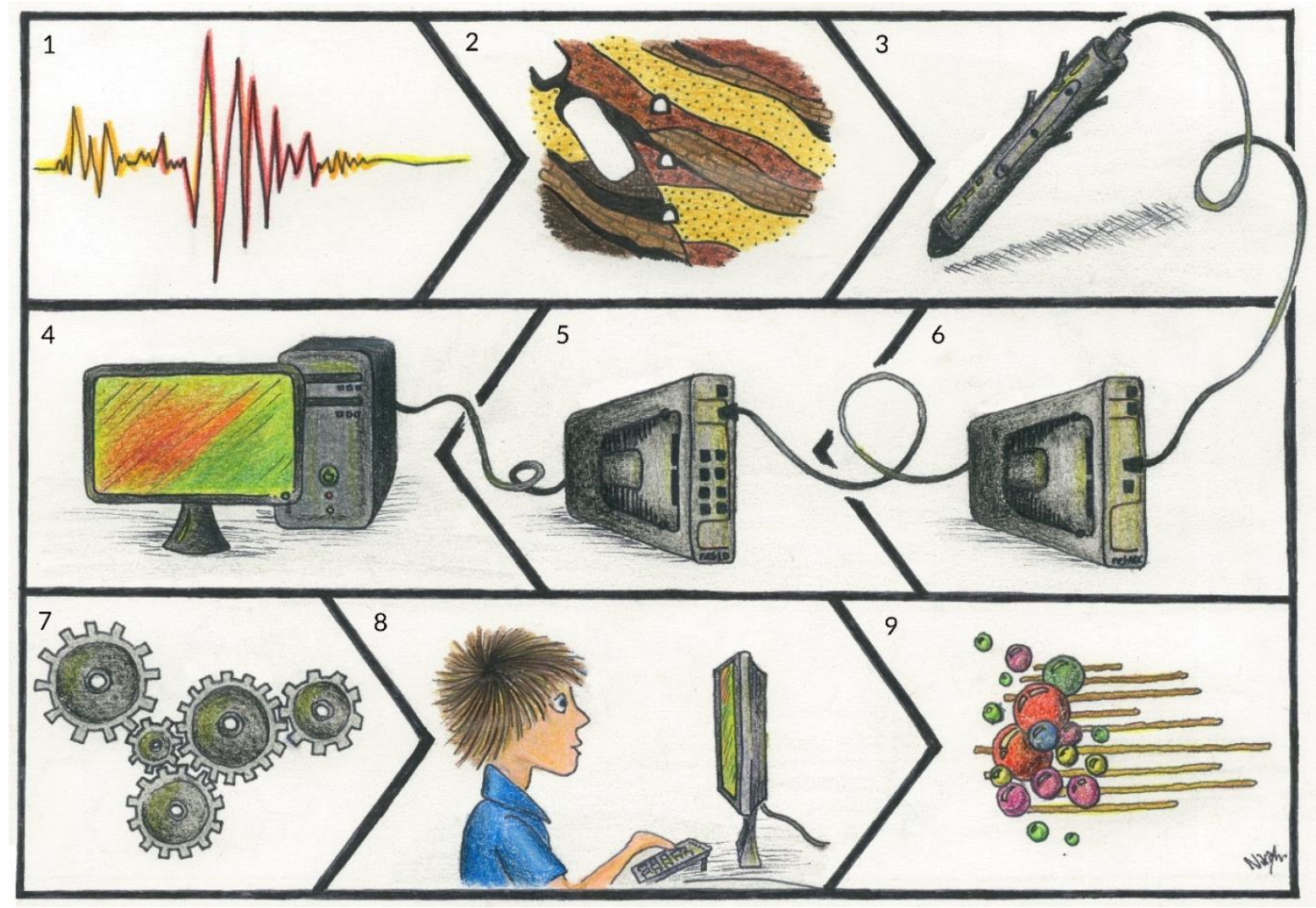

Figure 1 Flow diagram depicting the process involved when recording seismicity for a typical seismic system

Recently, Morkel et al. (2015) showed that seismic data for mines generally have several artefacts that contaminate the datasets. They showed that these artefacts might be due to noise (orepass and blasting), but that several other artefacts cannot be attributed to natural noise and were due to systematic errors. They are caused by abrupt changes in the way the seismic data was recorded, processed or source parameters calculated, and were not due to changes within the rock mass. They suggested that the majority of these artefacts are due to systematic inconsistencies. If this is true, a large portion of the artefacts could be eliminated by back processing the seismograms and ensuring the same location and source parameter calculation algorithms are consistently used for the whole database.

The Morkel and Wesseloo (2015) application implemented into mXrap (Harris \& Wesseloo 2015) considers several charts in an attempt to find artefacts in seismic datasets. Although this has been shown to be useful, it does still require some knowledge of seismic source parameters. Examples of the results of such investigations are shown in Morkel et al. (2015). We performed a general investigation into data consistency in mining induced databases, which indicated shifts in the data to be one of the biggest contributing factors to database inconsistency.

Therefore, although tools are available, it places unnecessary burden on the geotechnical engineers to hunt for these shifts and address them. Even more so to perform this task on an ongoing basis to ensure the problem is addressed as soon as it occurs.

A systematic and easily understandable technique is needed to find these shifts. Also, to quantify the extent of the problem, a methodical approach is required to quantify the severity of the problem. This paper proposes and discusses an easy to understand technique for determining when significant systematic shifts occur in seismic datasets. 


\section{Methodology}

This method is able to warn users of these changes as well as be computationally efficient. It is insensitive to the shape of the parameter distribution and uses a threshold value, necessary to trigger actions, which is easily understood by users and transferable to other databases.

The data used during this study are from several different mine seismic systems. These databases are current, and from seismic systems maintained and processed by several different institutions from countries across the world, including: Australia, Sweden, South Africa, the United States of America and Canada. The seismic parameters used in this study are:

- Seismic moment $\left(M_{w}\right)$.

- Seismic radiated energy (E).

- Corner frequency $\left(f_{0}\right)$.

- Apparent stress (AS).

- Apparent volume (AV).

- S-wave to P-wave energy ratio ( $\left.E_{s}: E_{p}\right)$.

For a description of the individual parameters see Gibowicz and Kijko (1994). Although this study focuses on these parameters, the technique is not limited to them. The parameters used are not all independent, but interdependency of the parameters does not adversely affect the technique.

The basic principle behind the technique is to compare chronological subsets of data statistically to evaluate when a significant shift has occurred. Initially, some established techniques were evaluated to determine when two chronological neighbouring sub-populations are significantly different. These included the two sample Kolmogorov Smirnov (KS test): chi-squared and z-test. These were applied over moving time-windows in an attempt to find the exact event in the database at which the shift occurs. Moving window calculations are performed over a chronologically sorted dataset. In this investigation, the size of the window is not defined by time but in the number of events. Applying a moving window to these techniques is a processing intensive operation. By design, these techniques give the same weight to smaller events in the distributions and are, therefore, sensitive to changes in seismic system sensitivity. Although such changes influence the database, they are not a result of the abrupt shifts being investigated.

Optimisation techniques that aim to obtain the best fit through subsets of data were also investigated. These techniques obtain the optimum number of groups by minimising the inter-group variation. They are less biased by smaller events but are processing intensive and, therefore, not practical for very large datasets, which is generally the case for microseismic databases.

The technique proposed in this paper evaluates the shifts in the mean of the different source parameters. To determine when abrupt shifts happen, the difference from the mean value for any event is determined by Equation (1).

$$
\Delta X_{i}=\frac{\sum_{i-N}^{i} X}{N}-\frac{\sum_{i+1}^{i+N+1} X}{N}
$$

where:

- $N$ is the half-size (number of events) of the block used.

- $i$ is the index of the event under question.

- $X$ is the parameter considered. 
This technique requires a single time window calculation for the whole database and is, therefore, efficient compared to the other methods investigated. Figure 2 illustrates the technique graphically. The blue circles in the figure indicate seismic events. For the example, the change in $\log \left(\mathrm{M}_{\mathrm{w}}\right)$ is investigated. For every event $i$, the difference between the mean value for an immediately historical (green arrow) and future time-window (orange arrow) is evaluated. The solid black line on the chart is the absolute value of that change. Peaks in this parameter coincide with shifts in the database.

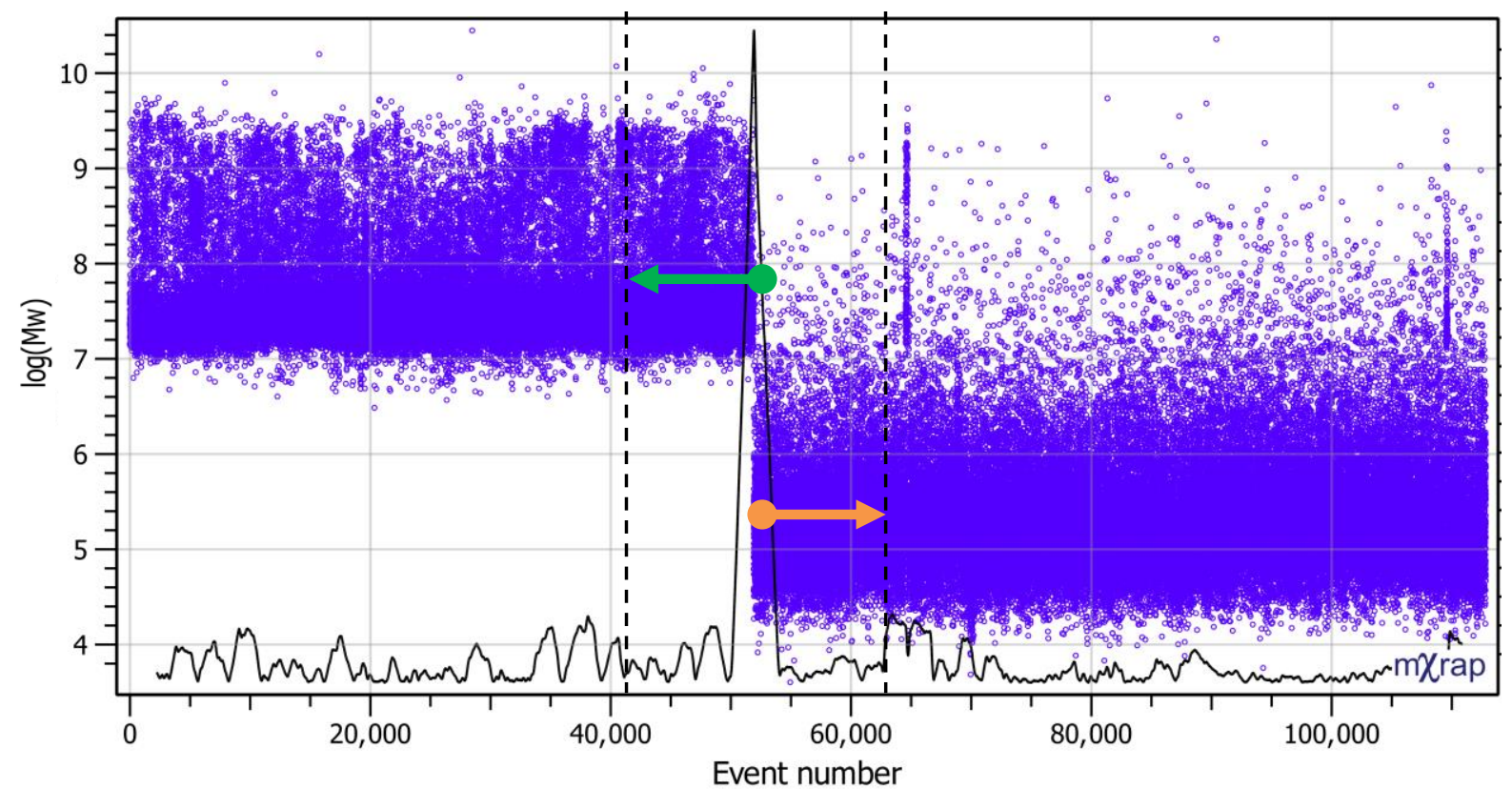

Figure 2 Illustration of the technique employed to determine when data shifts occur. The blue dots are the event data, the green arrow illustrates the historical mean and the orange the future mean. The black line is the absolute difference between the means for each event

This technique, requires the determination of an appropriate sample size ( $N$ in Equation (1)), and the definition of what constitutes a significant shift in the data. Each of the source parameters has different ranges and different associated threshold levels. The parameter's ranges are also site dependent and it is, therefore, important to define threshold values in a consistent manner across sites.

\subsection{Determination of sample size for moving window calculations}

To determine the sample size $(N)$, (Equation (1)) to use for each database, the deviation of the sub-sample mean to the database mean is investigated. This is done by randomly selecting events from the database and calculating the mean for that specific sample $(N)$. One expects the mean to converge to the database mean as more events are used to calculate the sub-sample mean. For each sample, the mean is calculated 100 times and these values are indicated through the symbols in Figure 3. The sample size that will be used for the calculations is where the variation from the database mean is less than $10 \%$ from the database mean within a $90 \%$ confidence envelope. Figure 3 shows an example of the convergence seen for the sub-sample means. The red line indicates the 90th percentile for all the source parameters. The density of data points is higher for lower percent deviation from the mean, resulting in the low apparent location of the 90th percentile line. Some of the values cannot be seen as they are hidden behind others. 


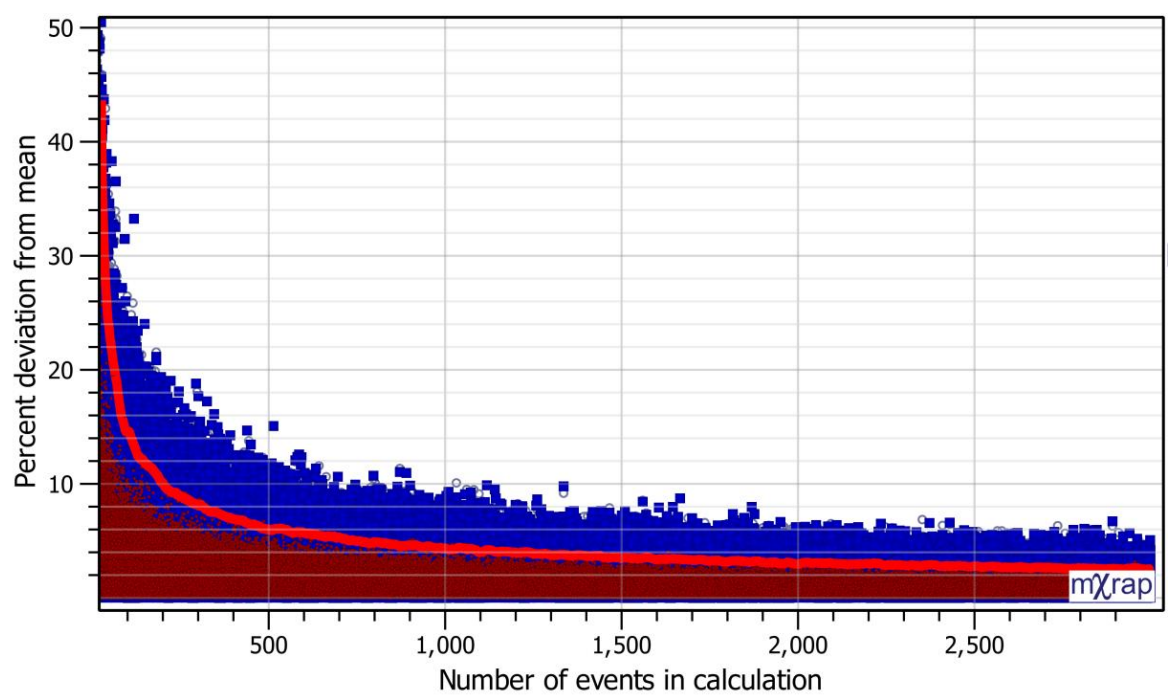

Figure 3 Percentage deviation from the sample mean for different sub-sample sizes. The coloured dots represent the calculated means, the grey crosses indicates the $\log (M w)$, grey circles the $\log (E)$, grey bars the $\log (\mathrm{fO})$, pink circles the $\log (\mathrm{S}: \mathrm{P})$, blue squares the $\log (\mathrm{AS})$, and red dots the $\log (\mathrm{AV})$. The red line indicates the 90th percentile for all the data combined

To ensure the shifts in data are not dominated by short term changes due to blasting, a lower limit of 500 events is used. Also, an upper limit of 2,500 events is used, as it was found that a larger time window smooths the data with subsequent loss in detail. These boundaries help to ensure the artefacts being searched for, are effectively highlighted.

\subsection{Determination of threshold values}

This technique also requires the determination of a transferable and easily understood threshold value. The threshold is expressed as the change of the mean of the data in terms of the standard deviation within the running time window. Standard deviation is found to be a good threshold value as most engineers have a good understanding of its meaning.

Changes with a value larger than the standard deviation are expected to correspond with the systematic shifts. Therefore, Equation (1) is normalised with standard deviation and becomes:

$$
\begin{gathered}
\Delta \tilde{X}_{i}=\left(\frac{\sum_{i-N}^{i} X}{N}-\frac{\sum_{i+1}^{i+N+1} X}{N}\right) / \sigma_{\min } \\
\sigma_{\min }=\min \left(\sigma_{-}-\sigma_{+}\right)
\end{gathered}
$$

where:

$$
\begin{aligned}
& \sigma_{-}=\text {standard deviation of } \mathrm{X} \text { for events } i-N \text { to } i . \\
& \sigma_{+}=\text {standard deviation of } \mathrm{X} \text { for events } i+1 \text { to } i+N+1 .
\end{aligned}
$$

The smallest standard deviation for the forward and backward half-blocks is used. This is to guard against a relaxation of the threshold triggering when a systematic change caused a reduction in the spread of data. This technique is performed for all of the source parameters and the largest value is retained for each event. The largest value is used rather than the stacked value to insure inter-parameter dependencies do not influence the results.

\subsection{Confirmation of statistical significance of flagged database shifts}

The shifts identified with Equation (2) are tested for similarity with the two sample KS tests. As mentioned before, performing KS tests on a running time window is process intensive. In this proposed methodology, the KS test is only applied on pre-determined shifts to confirm, with a high degree of certainty, the existence 
of those shifts. A confidence level of $99.99 \%$ is used throughout this study. When any of the parameters investigated is shown as being different from one sub-sample to the next, it is interpreted as confirming a shift in the database.

\section{$4 \quad$ Case study}

\subsection{Detailed evaluation of a database at a mine site}

This section provides a detailed discussion on the application of the presented technique on a seismic database from an underground mine. This database is the current working database for the mine at the time of writing this paper, and consists of approximately 40,000 events. The window size (N) used for this example is 1,500 events and a threshold value of $80 \%$ of $\sigma_{\min }$ is used to flag database shifts. The statistical significance of these shifts is confirmed via the two sample KS tests with a confidence level of $99.99 \%$.

The technique flagged six distinct groups which were confirmed to be significantly different with the KS test. The groups found by this technique are shown in Figure 4, with the sub-figures displaying the data scatter plots for the different source parameters. The different groups are shown with different colours.
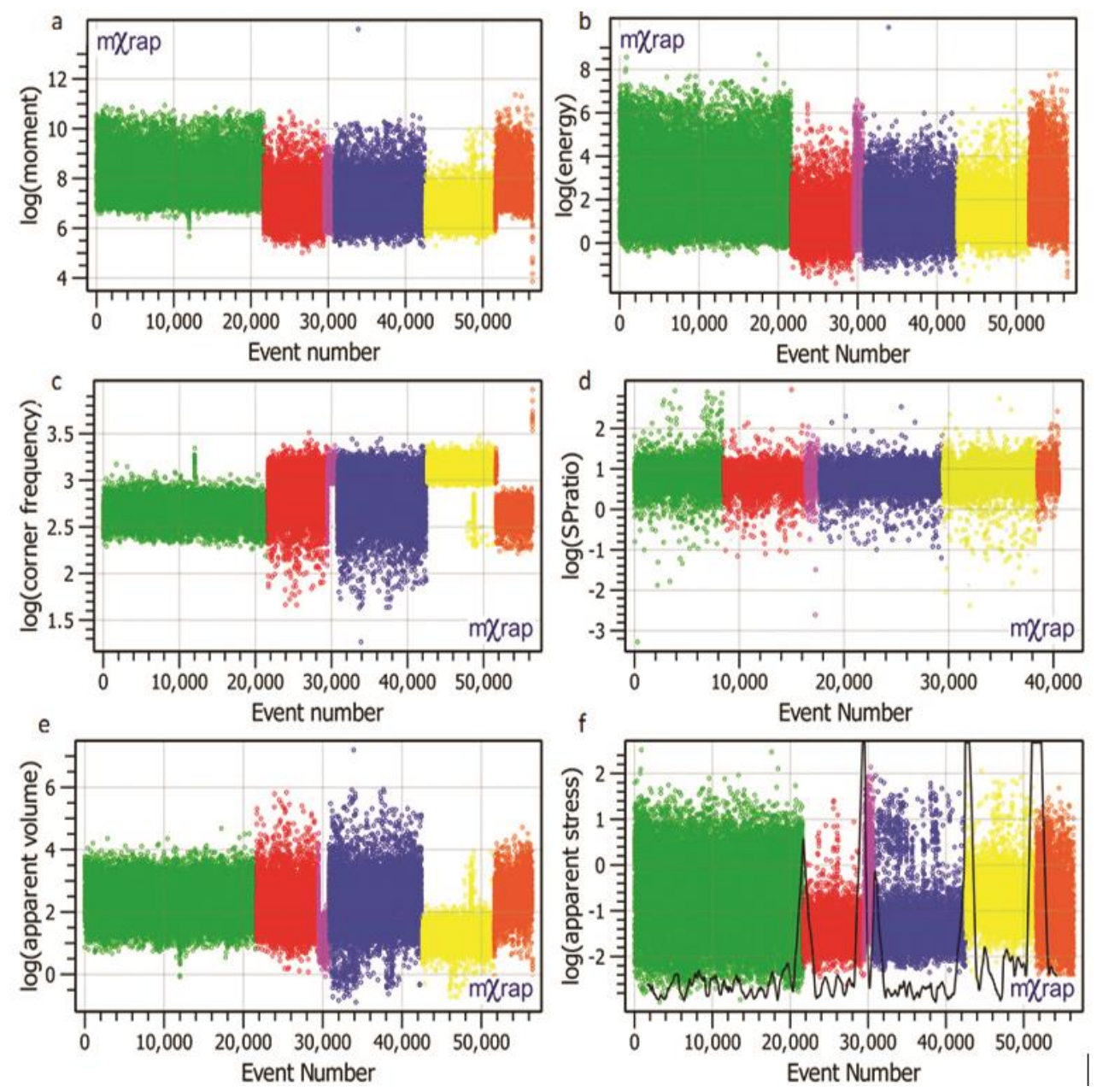

Figure 4 The different groups (coloured green, red, pink, blue, yellow and orange), as determined from the proposed technique. The six different parameters per event number is shown: (a) $\log \left(M_{w}\right) ;(b) \log (E) ;(c) \log \left(f_{0}\right) ;(d) \log (S:) ;(e) \log (A V) ;$ and, (f) $\log (A S)$

Table 1 lists the details of the shifts, as well as the results for the KS tests for each of the parameters. When the consecutive sub-groups are confirmed to be different by the KS test, it is highlighted in green. When the hypothesis is rejected, it is highlighted in red. The numbers for the KS test results are the difference between 
the KS-value and the $99.99 \%$ confidence value for the hypothesis. These numbers have no absolute meaning, but provide an indication of the relative significance of the shifts.

Table 1 Summary of the groups and the KS test results for each group

\begin{tabular}{lllllllll}
\hline Group \# & Up to date & $\begin{array}{l}\text { Up to } \\
\text { event \# }\end{array}$ & $\log \left(\mathrm{M}_{\mathrm{w}}\right)$ & $\log (\mathrm{E})$ & $\log \left(\mathrm{f}_{\mathbf{0}}\right)$ & $\log (\mathrm{S}: \mathrm{P})$ & $\log (\mathrm{AV})$ & $\log (\mathrm{AS})$ \\
\hline $0->1$ & $2007-09-18$ & 21,696 & 0.20 & 0.31 & 0.09 & 0.12 & 0.09 & 0.37 \\
\hline $1->2$ & $2008-01-01$ & 29,530 & 0.04 & 0.31 & 0.51 & 0.12 & 0.57 & 0.58 \\
\hline $2->3$ & $2008-01-23$ & 30,889 & -0.02 & 0.27 & 0.59 & 0.11 & 0.61 & 0.55 \\
\hline $3->4$ & $2008-07-08$ & 42,615 & 0.29 & 0.02 & 0.67 & 0.10 & 0.68 & 0.55 \\
\hline $4->5$ & $2008-11-12$ & 51,662 & 0.58 & 0.26 & 0.91 & 0.05 & 0.81 & 0.24 \\
\hline
\end{tabular}

Figure 5 shows the different groups on a $\log (E)-\log \left(M_{w}\right)$ chart and in Figure 6 the same groups are shown on a $\log \left(f_{0}\right)-\log \left(M_{w}\right)$ chart. These groups are the same as those shown in Figure 4, and the colours are consistently applied throughout the figures. It is clear that not only the parameter ranges for each group differ, but they differ to such an extent that it will influence the interpretation of the data. Hence, the conclusions made based on these parameters will not be trustworthy.

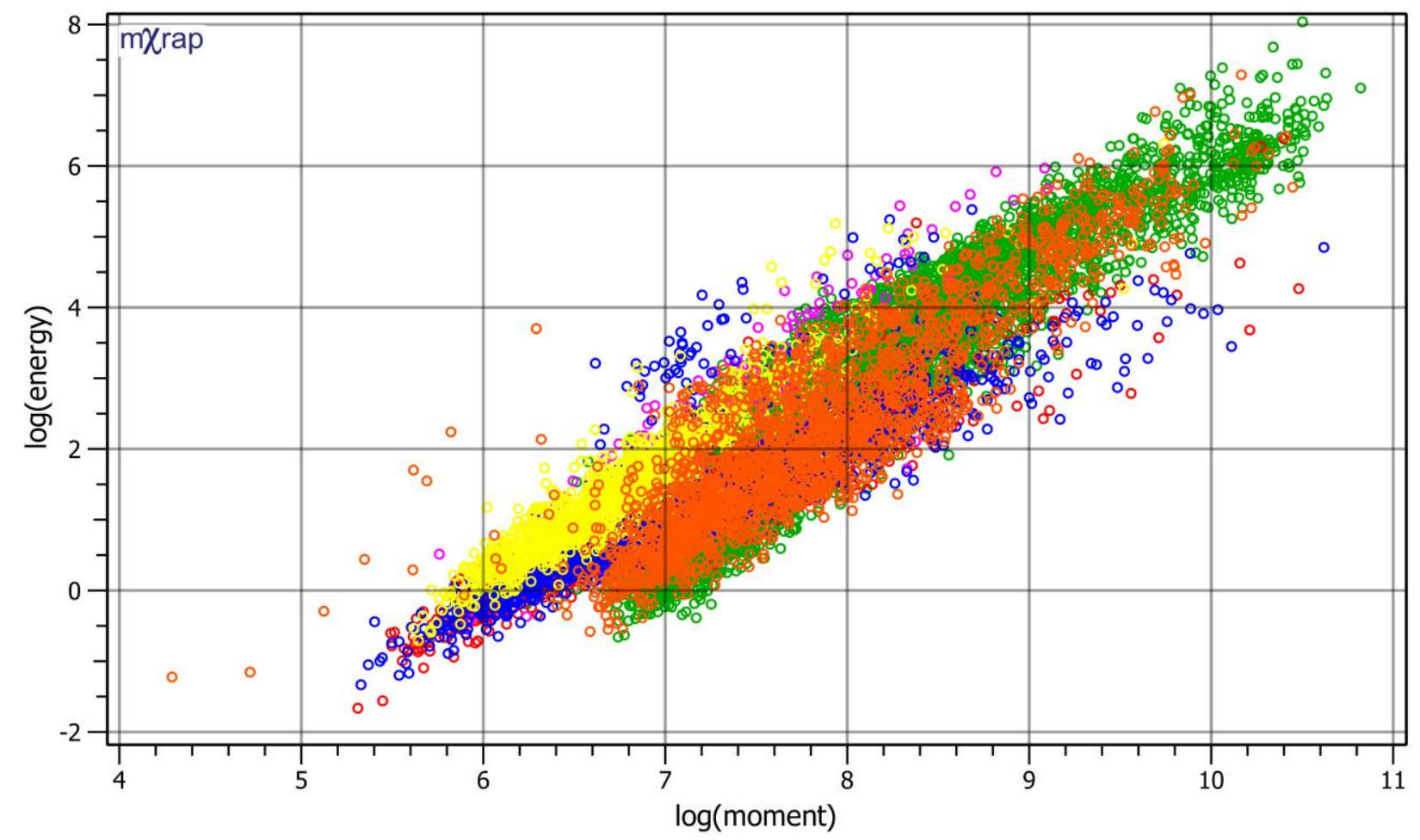

Figure $5 \log (\mathrm{E})$ versus $\log (\mathrm{Mw})$ chart for the different groups as determined by the proposed technique 


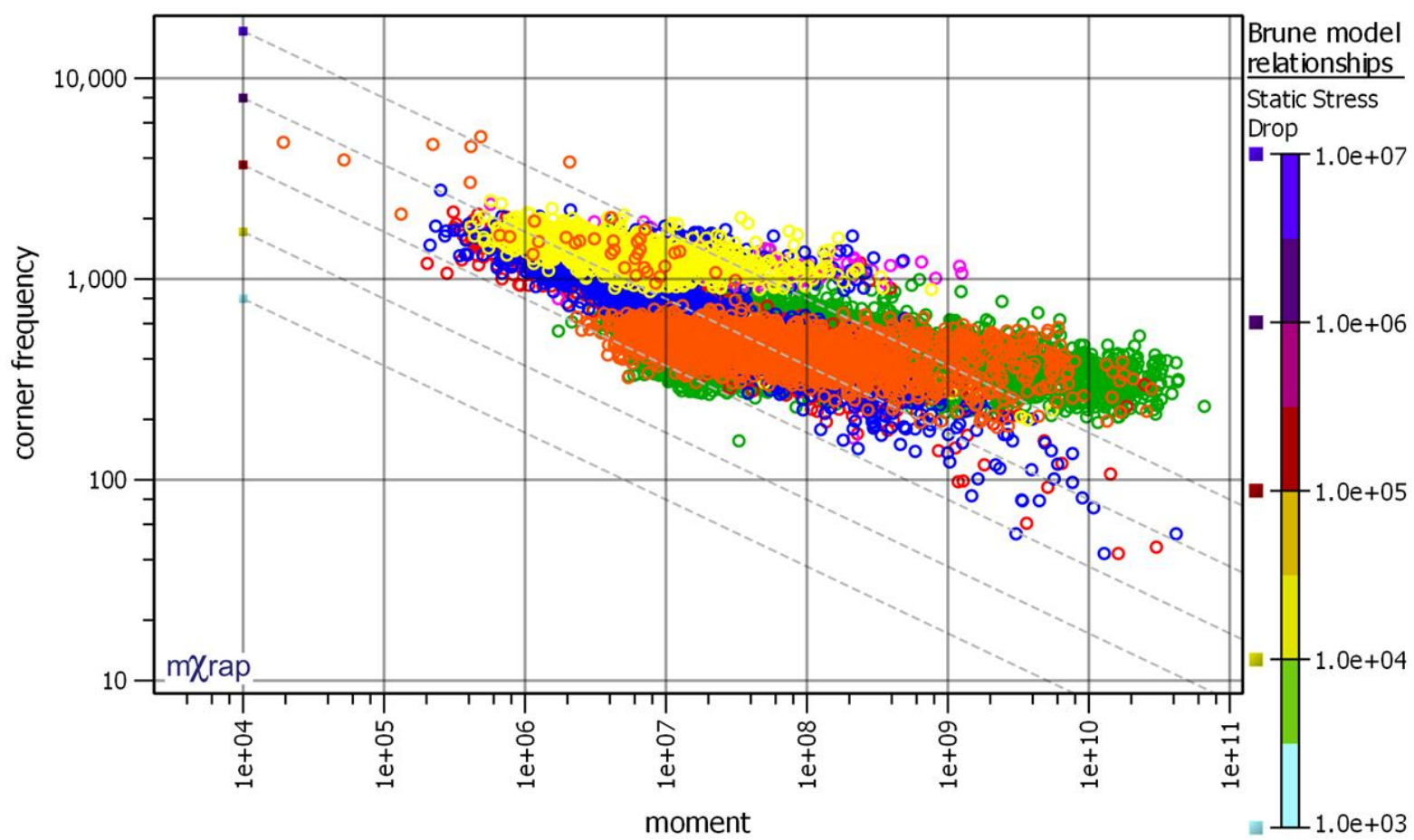

Figure $6 \log \left(f_{0}\right)$ versus $\log \left(M_{w}\right)$ chart for the different groups as determined by the proposed technique. The lines indicate the different static stress drop ranges

The $\log (E)-\log \left(M_{w}\right)$ relationship, for example, is used to determine the short term seismic hazard state by tracking the energy index over time (Rebuli \& Van Aswegen 2013; Van Aswegen 2005). The variations between the groups in Figure 6 mean that the basis of the energy index calculations differ for different periods of time. Any experience gained by engineers using the techniques proposed by Rebuli and Van Aswegen (2013) would be invalid after the date of shift in the database, and the interpretation would vary between different groups in the database. The differences in the groups (Figures 5 and 6), result in markedly different apparent stress values. The technique proposed by Brown (2015), Brown et al. (2015) would also suffer from the same limitations.

Such techniques would not be reliable for a database with systematic shifts in the data. We are not arguing that the technique is unreliable, but simply that such techniques cannot be applied reliably if consistency of the database is not ensured.

Figure 7 indicates the source radius calculated from the corner frequency for the different groups using Equation (3).

$$
R_{0}=\frac{2 \pi f_{0}}{k \cdot v_{s}}
$$

The source radius is based on the moment and static stress drop, as shown in Equation (4).

$$
R_{0}=\sqrt[3]{7 M_{0} / 16 S S D}
$$

where:

$$
\begin{aligned}
& R_{0}=\text { source radius. } \\
& f_{0}=\text { corner frequency. } \\
& k \quad=\text { parameter based on the displacement model. For the Brune } \omega^{2} \text {-model it is } 2.34 . \\
& v_{S}=\text { S-wave velocity. } \\
& M_{0}=\text { seismic moment. } \\
& S S D=\text { static stress drop. }
\end{aligned}
$$




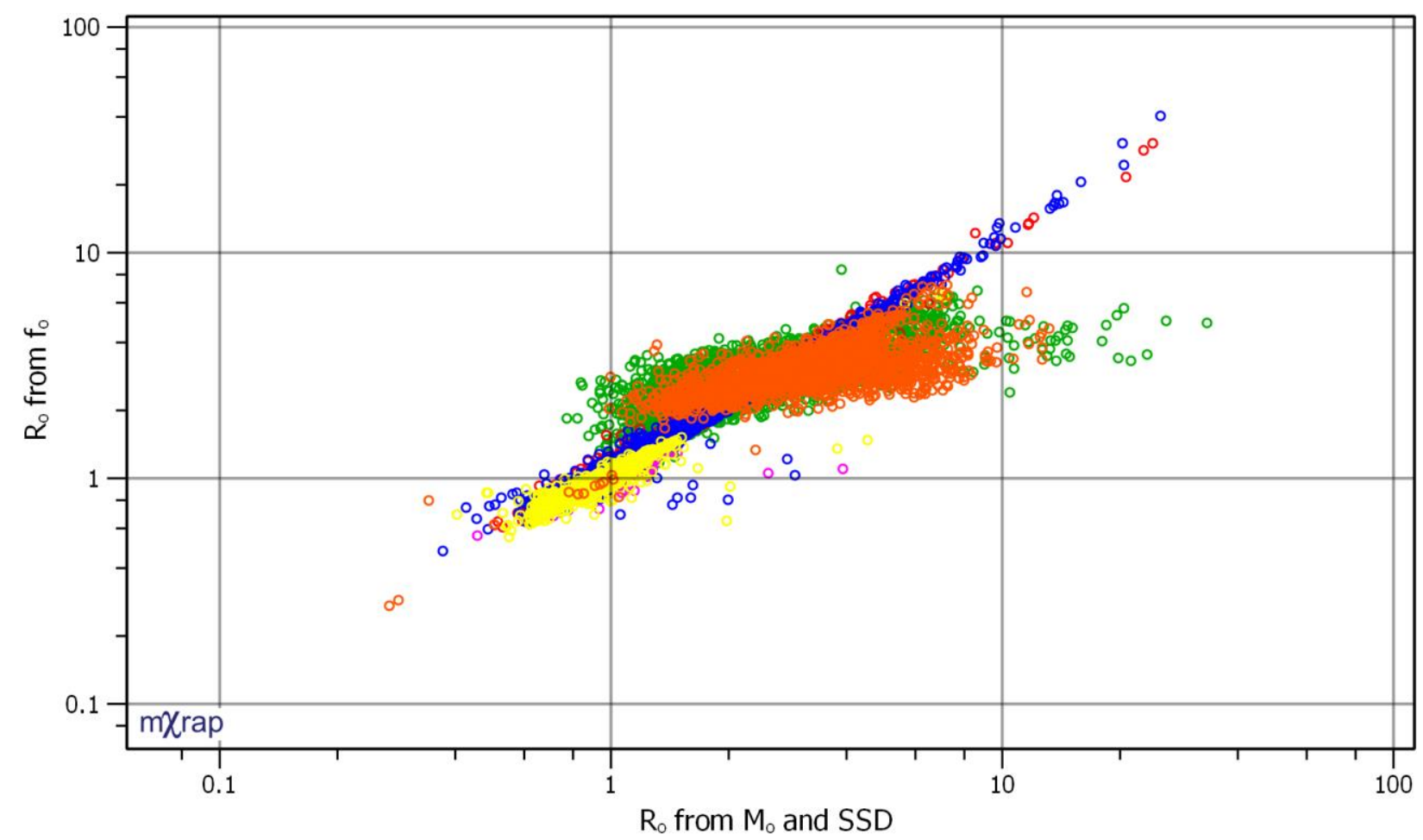

Figure 7 Comparison of the source radius for each event based on two different fundamental equations. On the $\mathrm{y}$-axis is the source radius based on the corner frequency, and on the $\mathrm{X}$-axis the source radius based on the seismic moment and static stress drop

One would expect variation of the data around a 1:1 line in Figure 7. Only some of the groups follow the expected trend while others have different slopes. These inter-parameter inconsistencies result from the shifts in the database and are of concern.

\subsection{Evaluation of the current working databases of several sites}

In the previous sections, the proposed technique was discussed and it was shown that the technique works well for detecting and quantifying statistically significant shifts in the dataset. In this section, the technique is applied to the databases of 20 sites around the world with different seismic system service providers.

For all of these databases, a conservative threshold value of $80 \%$ of the standard deviation was used to detect systematic shifts, and the KS test was employed at a confidence of $99.99 \%$ to confirm the statistical significance of these shifts. The results are expressed as the number of shifts per year and displayed in Figure 8 . Of the 20 sites, only one site did not have a statistically significant systematic shift. At several sites one statistically significant shift was detected in two years. However, $70 \%$ of the databases showed at least one significant shift per year, with some databases having up to five shifts a year. 


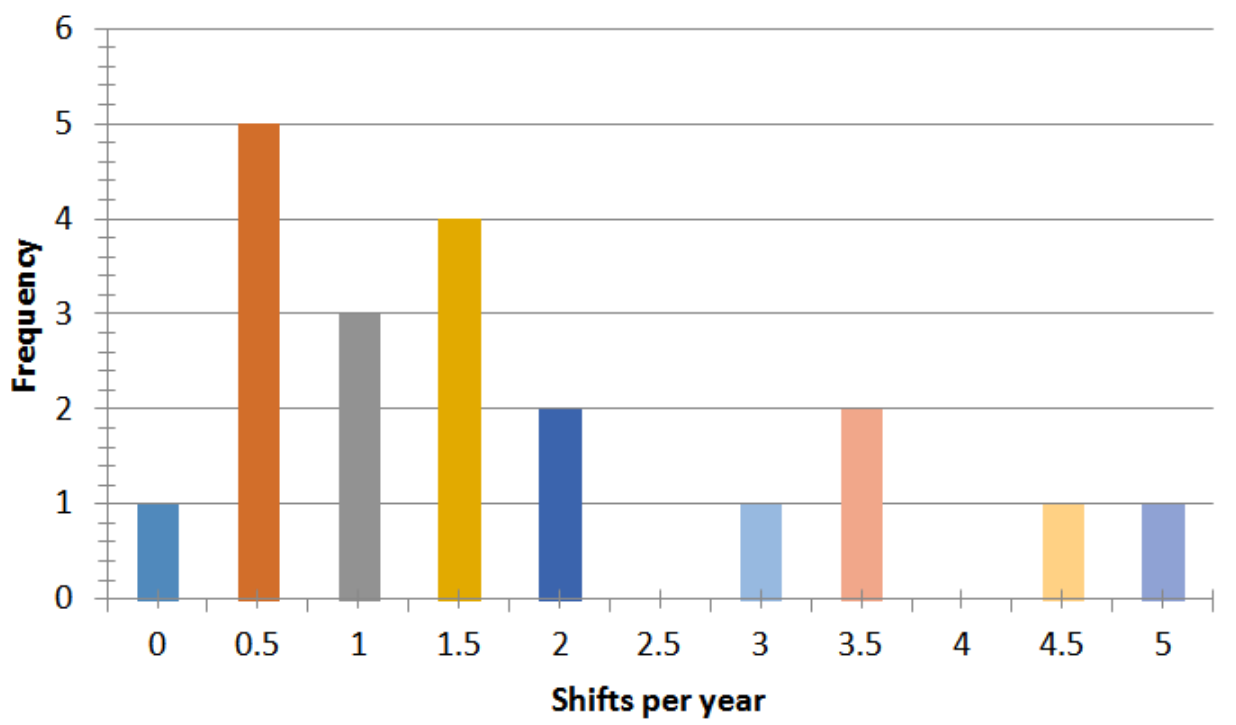

Figure 8 Histogram of the number of shifts per year for 20 databases

\section{Discussion}

The technique proposed in this paper works well and gives results that are consistent with expert investigation and has the following advantages:

- It is fast and processing friendly.

- It is expressed in such a way that the thresholds are understandable and easily communicated.

- The technique and the parameters are transferable between databases.

The technique was applied to current mine site databases and performed well in flagging and confirming statistically significantly different blocks of data, occurring due to systematic shifts in the database. The problem described in detail in Section 3 appears to be widespread and it appears that the importance of an internally consistent seismic database is not always appreciated and ensured. This confirms previous reports (Morkel et al. 2015).

Although the technique works well, it is reliant on changes in the mean value of the dataset. This means that in certain scenarios, for example where the mean does not change much but the variation is high, the proposed technique would not find a shift in the data. The technique is also dependent on abrupt sensitivity changes in the database, but less than the alternative statistical techniques.

As discussed in Sections 1 and 3, the shifts in some databases are sufficiently significant to render the use of some widely used analysis techniques unreliable. In the case study presented in this paper, it is shown that shifts in the data have a significant influence on the interpretation of the source parameters. The paper only discusses some examples, but many more analysis techniques that are heavily reliant on these parameters would also be affected. One of the bigger concerns is the shifts in both energy and moment. Such shifts would lead to different values for the local magnitude; determined from energy, moment or a combination of the two.

\section{Conclusion}

The proposed technique shows satisfactory performance and highlights the shift in source parameters. These shifts have been shown in a case study to be due to systematic errors in the datasets rather than gradual changes in the mining environment. This technique has its short comings; it can miss some shifts when the change is due to the spread of data, rather than a shift in the mean value. 
The results confirm the Morkel et al. (2015) suggestion that systematic errors are causing significant artefacts in seismic databases. Of the 20 databases investigated, $70 \%$ had one or more systematic shifts a year, and only one database showed no shifts at all.

This is a concerning result as such inconsistencies could lead to misinterpretation of seismic analysis results, which will have a carry-on effect on other parts of the operations. It is vital that these issues be addressed, and it is believed this could be dealt with by ensuring seismic databases are back processed whenever there are changes to the system.

The technique is fast and efficient and can, therefore, be easily employed on any database. By continuously updating results, users would know within a few tens to hundreds of events when data shifts have occurred. This would allow for the effective management of these errors in the database.

\section{Acknowledgement}

We acknowledge the support of the mXrap Consortium and the Australian Centre for Geomechanics during this project. Our sincere thanks to Anita-Marí Wesseloo for illustrating Figure 1.

\section{References}

Alcott, JM, Kaiser, PK \& Simser, BP 1998, 'Use of microseismic source parameters for rockburst hazard assessment', Pure and Applied Geophysics, vol. 153, pp. 41-65.

Brown, LG 2015, Seismic Hazard Evaluation Using Apparent Stress Ratio for Mining-Induced Seismic Events, Masters of Applied Science thesis, Laurentian University, Sudbury.

Brown, LG, Turcotte, P \& Hudyma, MR 2015, 'Seismic hazard assessment using apparent stress ratio', in Y Potvin (ed.), Proceedings of the International Seminar on Design Methods in Underground Mining, 17-19 November 2015, Perth, Australian Centre for Geomechanics, Perth, pp. 123-134.

Disley, NV 2014, 'Seismic risk and hazard management at Kidd Mine', in M Hudyma \& Y Potvin (eds), Proceedings of the Seventh International Conference on Deep and High Stress Mining, 16-18 September 2014, Sudbury, Australian Centre for Geomechanics, Perth, pp. 107-122.

Gibowicz, SJ \& Kijko, A 1994, An introduction to Mining Seismology, Academic Press, Inc.

Hanks, TC 1982, 'fmax', Bulletin of the Seismological Society of America, vol. 72, no. 6A, pp. 1867-1879.

Harris, PC \& Wesseloo, J 2015, mXrap, version 5, Australian Centre for Geomechanics, Perth, Western Australia, www.mXrap.com

Morkel, IG \& Wesseloo, J 2015, mXrap software app, Mining Induced Seismicity - Seismic Event Quality, version 1, Australian Centre for Geomechanics, The University of Western Australia, Perth, Western Australia, www.mXrap.com

Morkel, IG, Wesseloo, J \& Harris, PC 2015, 'Highlighting and quantifying seismic data quality concerns', in PM Dight (ed.), Proceedings of the Ninth International Symposium on Field Measurements in Geomechanics, 9-11 September 2015, Sydney, Australian Centre for Geomechanics, Perth, pp. 215-222.

Potvin, Y 2009, 'Strategies and tactics to control seismic risks in mines', Journal of the Southern African Institute of Mining \& Metallurgy, vol. 109, pp. 177.

Rebuli, DB \& Van Aswegen, G 2013, 'Short term seismic hazard assessment in S.A. gold mines', in A Malovichko \& D Malovicko (eds), Proceedings of the 8th International Symposium on Rockbursts and Seismicity in Mines, 1-7 September 2013, Saint Petersburg, Moscow, Geophysical Survey of Russian Academy of Sciences, pp. 323-331.

Scheepers, LJ, Hofmann, G \& Morkel, IG 2012, 'The study of seismic response to production for a grid mining layout', in W Joughin (ed.), Proceedings of the 2nd Southern Hemisphere International Rock Mechanics Symposium, 14-18 May 2012, Sun City, South African National Institute of Rock Engineering, pp. 387-406.

Van Aswegen, G 2005, 'Routine seismic hazard assessment in some South African mines', in Y Potvin \& M Hudyma (eds), Proceedings of the Sixth International Symposium on Rockburst and Seismicity in Mines, 9-11 March 2005, Perth, Australian Centre for Geomechanics, Perth, pp. 435-444.

Wesseloo, J 2013, 'Towards real-time probabilistic hazard assessment of the current hazard state for mines', in A Malovichko \& D Malovichko (eds), Proceedings of the 8th International Symposium on Rockbursts and Seismicity in Mines, 1-7 September 2013, Saint Petersburg, Moscow, Geophysical Survey of Russian Academy of Sciences, pp. 307-312.

Wesseloo, J 2014, 'Evaluation of the spatial variation of the b-value', Journal of the Southern African Institute of Mining and Metallurgy, vol. 114, pp. 823-828.

Wesseloo, J, Woodward, K \& Pereira, J 2014, 'Grid-based analysis of seismic data', Journal of the Southern African Institute of Mining and Metallurgy, vol. 114, no. 10, pp. 815-822. 